\title{
Gauss' Law and string-localized quantum field theory
}

\author{
Jens Mund, ${ }^{a}$ Karl-Henning Rehren ${ }^{b}$ and Bert Schroer ${ }^{c, d}$ \\ ${ }^{a}$ Departamento de Física, Universidade Federal de Juiz de Fora, \\ Campus Universitário da UJFJ, Juiz de Fora 36036-900, MG, Brasil \\ ${ }^{b}$ Institute for Theoretical Physics, Georg-August-University Göttingen, \\ Friedrich-Hund-Platz 1, 37077 Göttingen, Germany \\ ${ }^{c}$ Centro Brasileiro de Pesquisas Físicas, \\ Rua Dr. Xavier Sigaud 150, 22290-180 Rio de Janeiro, RJ, Brasil \\ ${ }^{d}$ Institut für Theoretische Physik, Freie Universität Berlin, \\ Arnimallee 14, 14195 Berlin, Germany \\ E-mail: jens.mund@ufjf.edu.br, krehren@gwdg.de, \\ schroer@zedat.fu-berlin.de
}

ABSTRACT: The quantum Gauss Law as an interacting field equation is a prominent feature of QED with eminent impact on its algebraic and superselection structure. It forces charged particles to be accompanied by "photon clouds" that cannot be realized in the Fock space, and prevents them from having a sharp mass $[7,19]$. Because it entails the possibility of "measurement of charges at a distance", it is well-known to be in conflict with locality of charged fields in a Hilbert space $[3,17]$. We show how a new approach to QED advocated in $[25,26,30,31]$ that avoids indefinite metric and ghosts, can secure causality and achieve Gauss' Law along with all its nontrivial consequences. We explain why this is not at variance with recent results in [8].

KeYwords: Gauge Symmetry, Global Symmetries, Space-Time Symmetries

ARXIV EPRINT: 1906.09596

Dedicated to Detlev Buchholz on the occasion of his 75th birthday. 


\section{Contents}

1 The quantum Gauss Law 1

1.1 Some history 1

1.2 Implications of the quantum Gauss Law 2

1.3 The string-localized formulation of QED 4

2 Bogoliubov's formula $\quad 6$

$\begin{array}{lll}3 & \text { An external field warm-up } & 7\end{array}$

4 Gauss' Law in string-localized QED $\quad 8$

$\begin{array}{lll}5 & \text { Infra-particles } & 10\end{array}$

6 The hybrid approach 13

$\begin{array}{lll}7 & \text { Photon cloud superselection } & 15\end{array}$

$\begin{array}{llr}8 & \text { Conclusions } & 18\end{array}$

$\begin{array}{lll}9 & \text { Outlook } & 19\end{array}$

A Some background material $\quad \mathbf{2 0}$

$\begin{array}{lll}\text { A.1 Vector potentials and indefinite metric } & 21\end{array}$

$\begin{array}{lll}\text { A.2 Causality and observables } & 23\end{array}$

\section{The quantum Gauss Law}

\subsection{Some history}

Before turning to the new results announced in the abstract, we want to call to mind some early developments and insights about the infrared intricacies of QED.

The message that interactions of photons with matter require a formulation which differs from standard scattering theory goes back to the work of Bloch and Nordsieck [2] in 1937 and Fierz and Pauli [18] in 1938. Decades later Yennie, Frautschi and Suura [36] integrated these early proposals into the covariant renormalized perturbation theory of QED. In this way the logarithmic on-shell infrared divergencies are encoded into the prescription for a rotational-invariant infrared photon inclusive cross-section for the scattering of charge-carrying particles. A closely related result of QED perturbation theory is (what is nowadays referred to as) Weinberg's soft photon theorem. 
As a matter of fact, apart from the rather indirect construction by Steinmann [32], up to date these heuristic on-shell prescriptions have not been supplemented by a direct construction of a physical ${ }^{1}$ Dirac field in causal perturbation theory. The present work is intended to report on substantial progress in this direction.

Back in the early 1960s, the LSZ scattering theory (which established the existence of in/out scattering states and the associated S-matrix as a consequence of the causal separability and the existence of a mass gap) suggested that what is at stake in QED is not only the large-time LSZ asymptotics, but the very concept of a Wigner particle with a sharp mass-shell. At that time the only indication for such an "infra-particle" without a sharp mass came from a model in $1+1$ spacetime dimensions by one of us [29], that also exhibited a non-trivial dynamical superselection structure.

More than another decade later, Ferrari, Picasso and Strocchi [17] proved that electrically charged fields cannot be pointlike localized, ${ }^{2}$ simply because local fields must commute with the charge operator, expressed as an integral over the electric field at spacelike infinity. Yet, it is indispensible for scattering theory that charged operators remain causally separable. Fröhlich, Morchio and Strocchi [19] showed that charged states cannot exist in the Fock representation and that the Lorentz symmetry is broken in irreducible charged representations. Buchholz [6] refined these insights by showing that there exists an uncountable set of superselection rules related to the asymptotic shape of photon clouds. Lorentz symmetry connects sectors with different such clouds. In the present paper we shall reveal an intimate relation between this fact of QED and the superselection structure in the model of [29] even if there were no gauge fields in that model. In a follow-up paper [7] Buchholz also proved that charge-carrying particles are necessarily infra-particles. The essential input was not perturbation theory but rather a careful formulation of the quantum Gauss Law.

In more recent times there has been a growing interest in large distance aspects related to interactions of massless fields of helicity $h \geq 1$ with $s<1$ matter fields, as documented in the extensive monograph on this subject [34]. A prominent issue is the existence of infinitely many "asymptotic symmetries", whose associated conservation laws are responsible for the asymptotic superselection structure.

\subsection{Implications of the quantum Gauss Law}

We present a brief review of the quantum Gauss Law (the measurement of the total electric charge at infinite spacelike distance and the ensuing failure of locality of charged fields) and its close relation to the uncountable set of "photon cloud" superselection rules. This cannot be understood in terms of the gauge-dependent point-localized matter fields of gauge theory that simply evade the conflict of the quantum Gauss Law with local commutation relations by a gauge fixing term [17], cf. appendix A.1, rather than address its physical consequences; instead we shall demonstrate how a new approach $[25,26,30,31]$ implements the many infrared features of QED without the need for state spaces with indefinite metric (Krein spaces) and ghosts to return to positive metric. In this approach, the physical interacting

\footnotetext{
${ }^{1}$ I.e., with positive definite correlation functions, cf. appendix A.1.

${ }^{2}$ Localization is always understood in the sense of causal commutation relations (vanishing commutator at spacelike distance). For some comments on "causality" and "observables", see appendix A.2.
} 
charge-carrying matter fields become "string-localized" via an interaction density coupling the point-localized free fields to "string-localized potentials". ${ }^{3}$

The Gauss Law allows to measure electric charges from a distance. The flux of the electric field across a closed surface $\sigma$ equals the total charge in the volume $V$ enclosed by the surface:

$$
\oint_{x^{0}=t, \vec{x} \in \sigma} d \vec{\sigma}(\vec{x}) \vec{E}(x)=\int_{x^{0}=t, \vec{x} \in V} d^{3} x j^{0}(x) .
$$

It follows from the inhomogeneous Maxwell equation (the differential Gauss Law)

$$
\partial_{\mu} F^{\mu \nu}(x)=j^{\nu}(x)
$$

In QED, the Maxwell field strength and the charge density are interacting quantum fields coupled through (1.2). The right-hand side equals $-q$ times the Dirac current, where the unit of charge $q$ is the coupling constant of QED.

The limiting case $V \rightarrow \mathbb{R}^{3}$, the global Gauss Law

$$
\lim _{R \rightarrow \infty} \oint_{x^{0}=t, \vec{x} \in R \cdot S^{2}} d \vec{\sigma}(\vec{x}) \vec{E}(x)=Q
$$

where the charge operator $Q$ generates the U(1) symmetry of the Dirac field, is particularly intriguing in the quantum theory: because the global charge operator is an integral over the field strength at spacelike infinity, and has a nontrivial commutator with the charged field, the latter cannot commute with the field strength at spacelike distance [3, 17].

This fact is only the first of a number of remarkable imprints of Gauss' Law on the algebraic structure of QED and the nature of charged particles, that are held to be characteristic of QED as a gauge theory [21].

The global charge operator and hence the asymptotic flux operator is a multiple of $\mathbf{1}$ in every irreducible representation of the algebra of local observables, defining the charge superselection rule. In contrast, local flux operators are dynamical quantities that are not central operators.

The global Gauss Law also requires that in charged states, the expectation value of the flux operator through a sphere of radius $R$ has a finite limit as $R \rightarrow \infty$. Hence, the field strength $F_{\mu \nu}(x)$ must decay in spacelike directions like $r^{-2}$, and the asymptotic values

$$
a_{\mu \nu}(x):=\lim _{\lambda \rightarrow \infty} \lambda^{2}\left(\Psi, F_{\mu \nu}(\lambda x) \Psi\right)
$$

are eigenvalues of central observables $\lim _{\lambda \rightarrow \infty} \lambda^{2} F_{\mu \nu}(\lambda x)$ in the irreducible representation of the state described by $\Psi$. Consequently, they define uncountably many "infrared" superselection rules. Lorentz symmetry connects different asymptotic field configurations (1.4) and can therefore not be realized in irreducible charged sectors [19]. Buchholz has shown in [7] (cf. section 5) that the condition of non-trivial limits in (1.4) along with a bound on the fluctuations entails that charged one-particle states cannot be eigenvectors of the mass

\footnotetext{
${ }^{3}$ A "string" is a spacelike or lightlike ray extending from a point to infinity. (In contrast, strings of String Theory are a classical concept that is not reflected in causal commutation relations.) String-localized approaches to QED were previously advocated by Mandelstam [22] and by Steinmann [32, 33].
} 
operator $M^{2}=P_{\mu} P^{\mu}$, hence charged particles cannot have a sharp mass, see section 5 . The effect can be ascribed to the "infrared photon clouds" attached to charged particles whose configuration is given by (1.4). This infra-particle nature of electrons is also responsible for the breakdown of the usual methods of scattering theory, discussed in section 1.1.

It should be clear from the previous, that the issues raised concern properties of the interacting fields themselves, and not just on-shell scattering amplitudes. The differential Gauss Law, constituting an algebraic relation between Maxwell and Dirac fields, is of course due to the interaction. The correct formulation of the interacting theory must be able to properly implement it.

\subsection{The string-localized formulation of QED}

In covariant quantizations of the Maxwell potential with indefinite metric, Gauss' Law does not hold (see appendix A.1). In the usual $\lambda$ gauges, there is a "fictitious current" $j_{\text {fict }}^{\mu}=$ $-\lambda \partial^{\mu}(\partial A)$, that is added to (1.2) as a contribution from the free field, and thus causes the global and local Gauss Laws to fail [3], cf. also section 4 and section 5. When the GuptaBleuler condition is imposed, the interacting Dirac field is no longer defined on the resulting physical Hilbert space, and charged states must be constructed in a different way, e.g., by a limit of charge separation [21]. An alternative to the Gupta-Bleuler method to deal with the indefinite metric is pursued in Steinmann's perturbative construction of QED [33].

In an emerging approach $[25,26,30,31]$, a perturbative construction of QED is proposed that avoids indefinite metric from the outset. Emphasizing fundamental principles of relativistic quantum theory, this approach aims at completing the successful Lagrangean construction of a unitary scattering matrix of QED by the construction of its Hilbert space fields with a full control of their causality properties. It starts from the fact (cf. appendix A.1), that the equation

$$
F_{\mu \nu}(x)=\partial_{\mu} A_{\nu}(x, e)-\partial_{\nu} A_{\mu}(x, e)
$$

can be solved (as a cohomological problem) by "string-localized potentials" [27]

$$
A_{\mu}(x, e):=\int_{0}^{\infty} d s F_{\mu \nu}(x+s e) e^{\nu},
$$

where $e$ is any (spacelike) direction in $\mathbb{R}^{4}$. The ray $\mathbb{R}_{+} \cdot e$ is referred to as "string" (cf. footnote 3). They are defined (as free fields) on the physical Hilbert space of the Maxwell field strength.

The perturbative expansion (cf. section 2) of QED with the interaction density

$$
L(x, e)=A_{\mu}(x, e) j^{\mu}(x)
$$

therefore proceeds on the tensor product of the free Maxwell and Dirac Hilbert spaces. Technically, the potential and $L(x, e)$ are distributions also in $e$ and will require a suitable smearing (that we suppress until section 7 ).

We show that in this approach, the inhomogeneous Maxwell equation (1.2) holds (already) in first perturbative order, and along with it the global and local Gauss Laws. 
We also show how the interacting Dirac field becomes string-localized, in accord with the NoGo result of [17]. (This is of course due to the string-localized interaction density; but the non-trivial part is to understand why the Dirac field is not worse than string-localized.)

We shall compute in the string-localized approach the asymptotic field configuration in states $\Psi=\psi^{*}(f) \Omega$, generated from the vacuum $\Omega$ by the smeared interacting Dirac field $\psi$. The remarkable feature is that — while the observable electromagnetic field strength remains string-independent - the unobservable charged field becomes string-dependent through the interaction with the string-localized potential, and in fact localized along the string. We find in this situation that the asymptotic electric flux in the string-dependent state is concentrated in the direction of the string $e$. Thus, choosing the direction $e$ (or an average over directions), one can generate states with "designed" photon clouds.

A recent proposal by Duch [12] constructing the interacting field strength by using a modified causal S-matrix in (2.1) below, also cures the global Gauss Law. Adapting old ideas of Dollard [11] and Kulish and Faddeev [16], it defines the S-matrix by comparison with a suitable (time-)asymptotic dynamics that takes the (space-)asymptotic photon clouds into account, corresponding to the "dressing transformations" of charged states in $[23,24]$. The localization of the interacting Dirac field is not addressed in that approach.

The findings presented here are not at variance with a recent result by Buchholz et al. [8]. The authors show (in a model with static charges, to be thought of as a limit case of QED with infinitely heavy charges) that it is impossible, with the help of only operators belonging to the algebra of the Maxwell field strengths, to generate states of that algebra in which the local flux operators take non-trivial values in accord with Gauss' Law. Namely, in the limit considered, the local flux operators are central elements of the algebra, and no approximations with inner conjugations can change their values. If the algebra is given (as is understood in [8]), an extension of the algebra is required. This can be conveniently achieved by potentials with "longitudinal degrees of freedom" that are not present in the field strengths. The string-localized potentials as in (1.5) cannot be used for the purpose.

In contrast, the construction of an interacting quantum field theory involves a change of the algebra of the free fields and of its representation. Perturbation theory achieves these changes. This is also true if the interaction density contains only observables, such as (1.6), because perturbation theory is not an approximation by inner unitary conjugations with degrees of freedom present in the interaction density. This is manifest in an external field problem (section 3), where perturbation theory is exact and the algebra remains unchanged, but the representation is manifestly changed: the local flux operators remain central but take the values required by the local Gauss Law.

The actual role of the string-localized potentials in the QED interaction density is to serve as "catalyzers" making the interacting Dirac fields string-localized, while the observable Maxwell fields remain point-localized. This remarkable structural resolution of the old locality issues of QED must have escaped the attention of the authors of [8] by their focus on the generation of charged states.

In the case of full QED with the string-localized interaction density (1.6), we restrict ourselves to first-order calculations. The results outlined before show that all the characteristic features of Gauss' Law in QED can be obtained in this way. In addition, we shall 
present a remarkable "semi-perturbative" formula, eq. (6.5), that reveals how the interacting Dirac field "virtually" (in a sense to be explained) carries the longitudinal degrees of freedom of the Maxwell potential of other approaches.

The full construction of the interacting charged field and the study of its large time asymptotic infra-particle scattering behaviour lie well beyond the scope of this paper.

\section{Bogoliubov's formula}

The perturbative approach is based on Bogoliubov's formula [4, Chap. 17] via a causal S-matrix functional of an IR cutoff function $g(x)$ for the coupling constant. The formula assigns to each free or composite free field $\chi$ an interacting field $\left.\chi\right|_{g L}$ where $L$ is the interaction density. This map deforms the algebraic relations, but it respects local commutativity of the interacting observables, thanks of the causal properties of the S-matrix functional. The renormalization is done in the Glaser-Epstein framework of causal perturbation theory.

Bogoliubov's formula with an interaction density $L(x)$ reads

$$
\left.\left.\int d^{4} x f(x) \chi\right|_{g L}(x) \equiv \chi\right|_{g L}(f):=-\left.i \partial_{\lambda} S[g, 0]^{*} S[g, \lambda f]\right|_{\lambda=0},
$$

where the causal S-matrix is the time-ordered exponential

$$
S[g, f]:=T e^{i \int d^{4} x[g(x) L(x)+f(x) \chi(x)]} .
$$

In first order,

$$
\left.\chi\right|_{g L}(x)=\chi(x)+\int d^{4} y g(y) R(L(y), \chi(x))+O\left(g^{2}\right),
$$

where $-i R(A(y), B(x)):=T[B(x) A(y)]-A(y) B(x)$ is the retarded commutator, and the higher order terms are multiple retarded commutators [13]. By the Wick expansion, the causal S-matrix $S[g, f]$ is expanded into a sum of Wick products times numerical retarded (anti-)commutators of free fields. Already these propagators are ill-defined by the prescription " $\theta\left(x^{0}-y^{0}\right)[\phi(x), \chi(y)]_{ \pm}$" (being products of distributions with overlapping singular supports), even more so are products thereof appearing in loop diagrams ill-defined. Their definition is a matter of renormalization and may introduce free parameters to be fixed by renormalization conditions. In Glaser-Epstein causal perturbation theory, renormalization is done in position space so as to keep control of localizations.

In the case of our interest, the interaction density (1.6) is string-localized, where $L(e)$ must be averaged with a test function $h(e)$. The renormalization is then subject to the "principle of string-independence": the S-matrix and observable fields must be independent of the auxiliary string variable $e$ or its averaging function $h$ in the "adiabatic limit" $g(x) \rightarrow q$.

The preservation of local commutativity of the observables is a crucial feature of Bogoliubov's formula. Let us sketch (in first order) how the argument proceeds, and why it fails for the non-observable Dirac fields in QED.

The commutator of two interacting fields $A_{1}, A_{2}$ in first order is

$$
\int d^{4} y g(y) C(y), \quad \text { where } \quad C(y)=\left[R\left(L(y), A_{1}\right), A_{2}\right]+\left[A_{1}, R\left(L(y), A_{2}\right)\right] .
$$


For sets $X, Y \subset \mathbb{R}^{4}$, we say that $X$ is later than $Y(X \succcurlyeq Y)$ if for each $x \in X$ and $y \in Y$, $x$ is not in the closure of the causal past of $y$. Two sets are spacelike separated iff $X \succcurlyeq Y$ and $Y \succcurlyeq X$. Let $X_{i}$ and $Y$ be the localizations of $A_{i}$ and $L(y)$, respectively. They may be points or strings.

Let the localizations $X_{i}$ of the free field operators $A_{i}$ be spacelike separated. If $Y \succcurlyeq X_{1}$ and $Y \succcurlyeq X_{2}$, then $R\left(L(y), A_{1}\right)=0=R\left(L(y), A_{2}\right)$ and hence $C(y)=0$. If $Y \nsucc X_{1}$ and $Y \nsucc X_{2}$, then the retarded commutators coincide with the ordinary commutators, and $C(y)=0$ by the Jacobi identity. For the other two cases, assume first that $Y=\{y\}$ is a point. If $y \succcurlyeq X_{1}$ and $y \nsucc X_{2}$, then $C(y)=i\left[A_{1},\left[L(y), A_{2}\right]\right]$. Now $y \nsucc X_{2}$, together with the fact that $X_{1} \succcurlyeq X_{2}$ implies by [10, Lemma 2.5] that $X_{1} \succcurlyeq y$, and because also $y \succcurlyeq X_{1}, y$ is spacelike separated from $X_{1}$, and $C(y)=0$ because $A_{1}$ commutes with $L(y)$ and with $A_{2}$. Similar for $y \succcurlyeq X_{2}$ and $y \nsucc X_{1}$. Hence $C(y)=0$ for all $y$. Thus, a point-local interaction density preserves local commutativity of string-localized fields.

Lemma 2.5 in [10] does not apply when $Y$ is a string $y+\mathbb{R}_{+} e$, even if $X_{i}$ are points. But when $A_{i}(x)$ are observable fields, which, by definition, remain independent of $e$ under the interaction, one can exploit the option to choose $e$ appropriately, so that the same conclusion holds: commutativity of point-local observables is preserved by string-local interactions. We shall present an alternative, more elegant argument in section 6 .

For non-observable fields, one has to expect an uncontrolled delocalization under a string-localized interaction, in general. However, for the interacting Dirac field in QED, we shall present a remarkable formula in section 6 by which it behaves like a string-localized field under a point-localized interaction. Thus, it "inherits" the string-localization of the interaction density. This is in accord with the NoGo result of [17] mentioned in the introduction. If $e$ is chosen spacelike, this also ensures sufficient spacelike separability for the needs of scattering theory.

\section{An external field warm-up}

We present a simple example, where perturbation theory is exact. The example is the external field problem with $\mathcal{L}_{h}=F^{\mu \nu} h_{\mu \nu}$ with an arbitrary classical source $h_{\mu \nu}(x)$. We shall show that the Bogoliubov map changes the values of the central flux operators. This shows that, even when the interaction density is a functional of the observables, the Bogoliubov map is not an approximation by inner conjugations.

In the case at hand, Bogoliubov's formula (2.1) simplifies to

$$
\int d^{4} x F_{h}^{\mu \nu}(x) f_{\mu \nu}(x) \equiv F_{h}(f)=-\left.i \partial_{\lambda} S[h]^{*} S[h+\lambda f]\right|_{\lambda=0}
$$

where

$$
S[h]:=T e^{i \int d^{4} x \mathcal{L}_{h}(x)}=T e^{i F(h)} .
$$

(As the external field $h$ plays the role of the cutoff function $g$, an adiabatic limit is not taken.)

The retarded propagator

$$
R(F(f), F(h))=G_{\mathrm{ret}}(f, h) \cdot \mathbf{1} \equiv \int d^{4} x d^{4} y f_{\mu \nu}(x) h_{\kappa \lambda}(y) G_{\mathrm{ret}}^{\mu \nu, \kappa \lambda}(x-y) \cdot \mathbf{1}
$$


is given in terms of the massless scalar propagator $G_{\mathrm{ret} 0}(z)=-\int \frac{(2 \pi)^{-4} d^{4} k}{k^{2}+i \varepsilon k^{0}} e^{-i k z}$ :

$$
G_{\text {ret }}^{\mu \nu, \kappa \lambda}(z)=\partial^{[\mu} \eta^{\nu][\lambda} \partial^{\kappa]} G_{\text {ret } 0}(z)
$$

(the brackets denoting anti-symmetrization). Thus, standard use of Wick's theorem implies

$$
S[h]=e^{\frac{i}{2} G_{\mathrm{ret}}(h, h)} \cdot e^{i F(h)}
$$

and

$$
S[h]^{*} S[h+f]=e^{i G_{\mathrm{ret}}(f, h)+\frac{i}{2} G_{\mathrm{ret}}(f, f)} \cdot e^{i F(f)} .
$$

Bogoliubov's formula then yields

$$
F_{h}(f)=F(f)+G_{\mathrm{ret}}(f, h) \cdot \mathbf{1}, \quad \text { or } \quad F_{h}^{\mu \nu}(x)=F^{\mu \nu}(x)+\int d^{4} y G_{\mathrm{ret}}^{\mu \nu, \kappa \lambda}(x-y) h_{\kappa \lambda}(y) \cdot \mathbf{1} .
$$

The string-localized interaction density $A^{\mu}(x, e) j_{\mu}(x)$ with a classical conserved source $j_{\mu}(x)$ is a special case, because the action can be written as

$$
\int d^{4} x A^{\mu}(x, e) j_{\mu}(x)=F\left(h^{e}\right),
$$

where $h_{\mu \nu}^{e}(x)=\frac{1}{2} \int_{0}^{\infty} d s j_{[\mu}(x-s e) e_{\nu]}$.

When the Maxwell Green function is expressed in terms of the scalar Green function, the shift term becomes

$$
\left(G_{\mathrm{ret}} h^{e}\right)^{\mu \nu}(x) \equiv \int d^{4} y G_{\mathrm{ret} 0}(x-y) \eta^{\kappa[\mu} \partial^{\nu]} \partial^{\lambda} \int_{0}^{\infty} d s j_{[\kappa}(y-s e) e_{\lambda]} .
$$

Because $\partial^{\lambda} j_{\lambda}=0$ and $e_{\lambda} \partial^{\lambda} j(y-s e)=-\partial_{s} j(y-s e)$, the derivative $\partial^{\lambda}$ of the $s$-integral just yields $j_{\kappa}(y)$, which is independent of $e$. Actually, the retarded propagator of the field strength is unique only up to a term $c \cdot\left(\eta^{\mu \kappa} \eta^{\nu \lambda}-\eta^{\nu \kappa} \eta^{\mu \lambda}\right) \delta\left(x-x^{\prime}\right)$. This term would add a contribution to (3.3) that depends on $e$. The principle of $e$-independence fixes the renormalization constant $c=0$.

Thus, the shift term is independent of $e$ and equals the classical retarded electromagnetic field with source $j^{\mu}$,

$$
F_{\text {class }}^{\mu \nu}(x)=\int d^{4} y G_{\text {ret } 0}(x-y) \partial^{[\nu} j^{\mu]}(y) .
$$

In particular, the flux operator is shifted by the classical value of the flux.

No unobservable quantum degrees of freedom are needed to achieve this result. This instance is in contrast to the statement in the abstract of [8], that "[gauge] bridges are needed in order to ensure the validity of Gauss' law".

\section{Gauss' Law in string-localized QED}

The following explicit first-order calculation of the interacting flux operator in stringlocalized QED shows that Bogoliubov's formula changes the free flux operators to interacting flux operators that satisfy Gauss' Law as an operator equation, with the electric 
charge operator "on the right-hand side" plus a boundary term that weakly vanishes in the adiabatic limit.

The string-localized interaction density is $L(x, e)=A_{\mu}(x, e) j^{\mu}(x)$, where $j^{\mu}=: \bar{\psi} \gamma^{\mu} \psi:$ is the Dirac current. We choose for simplicity $e=(0, \vec{e})$.

The interacting field strength in first order is

$$
\left.F_{\mu \nu}\right|_{g L(e)}(x)=F_{\mu \nu}(x)+\int d^{4} y g(y) R\left(A_{\kappa}(y), F_{\mu \nu}(x)\right) j^{\kappa}(y)+O\left(g^{2}\right) .
$$

The retarded commutator arises from $R\left(F_{\kappa \lambda}(y+s e), F_{\mu \nu}(x)\right) e^{\lambda}$ by distributional integration over the string according to $(1.5) . R(F, F)$ is unique up to a renormalization as in section 3. The choice $c=0$ yields

$$
R^{(0)}\left(A_{\kappa}(y), F_{\mu \nu}(x)\right)=-\left(\partial_{[\mu}^{x} \eta_{\nu] \kappa}+\partial_{[\mu}^{x} e_{\nu]} \partial_{\kappa}^{y} I_{e}^{y}\right) G_{\mathrm{ret} 0}(x-y) \cdot \mathbf{1},
$$

where $\left(I_{e}^{y} f\right)(y):=\int_{0}^{\infty} d s f(y+s e)$ is the string integration operator with inverse $-\left(e \partial_{y}\right)$.

Its divergence is

$$
\partial_{x}^{\mu} R^{(0)}\left(A_{\kappa}(y), F_{\mu \nu}(x)\right)=\left[-\eta_{\nu \kappa} \delta(x-y)+e_{\nu} \partial_{\kappa}^{x} \int_{0}^{\infty} d s \delta(x-y-s e)\right] \cdot \mathbf{1} .
$$

Thus,

$$
\left.\partial^{\mu} F_{\mu \nu}\right|_{g L(e)}(x)=-g(x) j_{\nu}(x)+e_{\nu} \int_{0}^{\infty} d s \partial_{\kappa} g(x-s e) \cdot j^{\kappa}(x-s e)+O\left(g^{2}\right) .
$$

The first term becomes $-q j_{\nu}(x)$ in the adiabatic limit. For spacelike $e$, the second term, evaluated in an electron state $\Psi=\psi^{*}(f) \Omega,\|\Psi\|^{2}=1$, goes to zero in the adiabatic limit, because as the region where $g(x)=q$ increases, the support of $\partial_{\kappa} g(x)$ moves to infinity in a spacelike direction, where $\left(\Psi, j^{\kappa}(x) \Psi\right)$ decays rapidly. Thus, the adiabatic limit exists in the weak sense, and the differential Gauss Law holds weakly. In the adiabatic limit,

$$
\int_{V} d^{3} x\left(\Psi,\left.\vec{\nabla} \vec{E}\right|_{q L(e)}(0, \vec{x}) \Psi\right)=-q \int_{V} d^{3} x\left(\Psi, j_{0}(0, \vec{x}) \Psi\right)+O\left(q^{2}\right) .
$$

If the volume $V$ is large enough that its complement is spacelike separated from the support of $f$, this equals $-q$. In particular, also the global Gauss Law (1.3) holds.

Because $\partial_{\mu} F^{\mu \nu}=0$ in 0th order, the 1st order corrections of $\left.\psi(f)\right|_{q L(e)}$ do not contribute, and the previous are the full first order results. Higher perturbative orders are needed to turn the current on the right-hand side into the interacting current.

If the same calculations leading to (4.2) were done in the point-local indefinite-metric (Krein space) setting, for simplicity in the Feynman gauge $\lambda=1$, the zeroth-order term $\partial^{\mu} F_{\mu \nu}=-\partial_{\nu}(\partial A)$ would not vanish (the "fictitious current" mentioned in section 1.3, cf. appendix A.1) and must be added to (4.2). We shall see in section 5 that the fictitious current contributes to the expectation value of $\partial_{\mu} F^{\mu \nu}$ in states generated from the vacuum by the interacting Dirac field, so as to cancel the global charge. In view of [17], this is a necessity because the Dirac field is point-localized in indefinite-metric Feynman gauge QED, and consequently commutes with the gobal charge operator. Conversely, in the 
string-localized approach, the global charge is the expected one, and the charged fields are string-localized.

The bulk term $-g(x) j(x)$ in (4.2) would be the same in the indefinite-metric setting, but the boundary term would be instead

$$
\int d^{4} y g(y) j^{\kappa}(y) \partial_{\kappa} \partial_{\nu} G_{\mathrm{ret} 0}(x-y)=\int d^{4} y \partial_{\kappa} g(y) \cdot j^{\kappa}(y) \partial_{\nu} G_{\operatorname{ret} 0}(x-y) .
$$

This vanishes for large spacelike $x$ where the support of $G_{\text {ret0 }}(x-y)$ does not intersect the support of $\partial g(y)$. If the integral of the zero-component over the $x^{0}=0$-plane is computed before the adiabatic limit is taken, the integral over $\partial_{0} G_{\text {ret } 0}(x-y)$ yields $\theta\left(x^{0}-y^{0}\right)$, and by partial integration the boundary term (4.4) exactly cancels the bulk term. On the other hand, the decay of (4.4) in the adiabatic limit for finite $x$ is harder to control because the integration extends over the intersection of the support of $\partial g$ with the entire backward lightcone of $x$ : the concentration of the boundary term along the string in (4.2) is a technical advantage of the string-localized approach.

The propagator of the field strength, and consequently also the retarded commutator, has a renormalization freedom

$$
R^{(c)}\left[F_{\mu \nu}(x), F_{\kappa \lambda}(y)\right]=R^{(0)}\left[F_{\mu \nu}(x), F_{\kappa \lambda}(y)\right]+c \cdot\left(\eta_{\mu \kappa} \eta_{\nu \lambda}-\eta_{\nu \kappa} \eta_{\mu \lambda}\right) \delta(x-y) \cdot \mathbf{1} .
$$

Integration over the string $e=(0, \vec{e})$ gives the corresponding freedom for the retarded commutator $R\left(A_{\kappa}(y), F_{\mu \nu}(x)\right)$ of the electric field with the string-localized potential

$$
-i c \cdot \eta_{\kappa[\mu} e_{\nu]} \int_{0}^{\infty} d s \delta(x-y-s e) .
$$

Its divergence is $c$ times (4.1). Thus, the renormalization freedom just renormalizes the electric charge. In other words, if $q$ is the physical unit of charge, then one must choose $c=0$, cf. also [24]. The same choice is also dictated by the principle of string-independence (as in section 2).

\section{$5 \quad$ Infra-particles}

In order that the global charge operator $=$ electric flux through the infinite sphere exists and is non-zero in charged states $\Psi$, the field strength should decay like $r^{-2}$ in spacelike directions (corresponding to the classical Coulomb Law). Thus, for the interacting field the limit

$$
f_{\mu \nu}(x):=\lim _{\lambda \rightarrow \infty} \lambda^{2} F_{\mu \nu}(\lambda x)
$$

for spacelike $x$ should exist in the weak sense (matrix elements), be non-zero and have finite fluctuations in charged states [7]. Because $f_{\mu \nu}(x)$ commutes with all local observables, it is a multiple of $\mathbf{1}$ in every irreducible representation: $f_{\mu \nu}(x)=a_{\mu \nu}(x) \cdot \mathbf{1}$. This asymptotic field configuration $a_{\mu \nu}(x)$ is by construction a homogeneous function of $x$ of degree -2 . Buchholz [7] has shown that these properties imply that charged states cannot be eigenvectors of the mass operator $M^{2}=P_{\kappa} P^{\kappa}$. (For a simplified argument, see appendix A.2.) 
We conclude that the electrons of QED are "infra-particles" (particles without a sharp mass), because Gauss' Law enforces the decay like $r^{-2}$ of the surrounding electromagnetic field (the "photon cloud"). In the two-point function of fields describing infra-particles, the $\delta$ function $\delta\left(p^{2}-m^{2}\right)$ is replaced by some continuous function with a singularity (cf. the 2D model in [29]). This in turn entails that correlations decay faster in asymptotic time than with a sharp mass. For this reason, the scattering theory for infra-particles needs a nontrivial adjustment of the LSZ formalism.

We want to verify the infra-particle features of QED in the string-localized approach. The validity of the global Gauss Law established in section 4 already entails the $\lambda^{-2}$ decay of the radial electric field (flux density). We want to calculate also its directional distribution.

To this end, we have to evaluate the asymptotic field configuration of the interacting field in charged states $\left.\Psi\right|_{g L(e)}:=\left.\psi^{*}\right|_{g L(e)}(f) \Omega$ created by the interacting Dirac field:

$$
a_{\mu \nu}(x)=\left.\lim _{\lambda \rightarrow \infty} \lambda^{2}\left(\Psi, F_{\mu \nu}(\lambda x) \Psi\right)\right|_{g L(e)}
$$

In contrast to indefinite-metric approaches, this state defines a positive functional.

The three first-order contributions to $\psi, \bar{\psi}$ and $F$ in $\left\langle\psi(f) F^{\mu \nu}(x) \psi(f)^{*}\right\rangle$ are in turn

$$
\begin{aligned}
X_{1} \equiv\left\langle\psi^{(1)}(f) F_{\mu \nu}(x) \psi(f)^{*}\right\rangle & =\int d^{4} y g(y)\left\langle R\left(j^{\kappa}(y), \psi(f)\right) \psi(f)^{*}\right\rangle \cdot D_{\kappa, \mu \nu}^{y} \Delta_{0}(y-x), \\
X_{2} \equiv\left\langle\psi(f) F_{\mu \nu}(x) \psi^{(1)}(f)^{*}\right\rangle & =\int d^{4} y g(y)\left\langle\psi(f) R\left(j^{\kappa}(y), \psi(f)^{*}\right)\right\rangle \cdot D_{\kappa, \mu \nu}^{y} \Delta_{0}(x-y), \\
X_{3} \equiv\left\langle\psi(f) F_{\mu \nu}^{(1)}(x) \psi(f)^{*}\right\rangle & =\int d^{4} y g(y)\left\langle\psi(f) j^{\kappa}(y) \psi(f)^{*}\right\rangle \cdot D_{\kappa, \mu \nu}^{y} G_{\text {ret } 0}(x-y),
\end{aligned}
$$

where $\Delta_{0}$ and $G_{\text {ret0 }}$ are the massless scalar 2-point functions and retarded propagator. The tensor of integro-differential operators

$$
D_{\kappa, \mu \nu}^{y}=\left(\partial_{\mu}^{y} \eta_{\nu \kappa}-\partial_{\nu}^{y} \eta_{\mu \kappa}\right)+\partial_{\kappa}^{y}\left(\partial_{\mu}^{y} e_{\nu}-\partial_{\nu}^{y} e_{\mu}\right) I_{e}^{y}
$$

arises from the two-point functions involving $F_{\mu \nu}(x)$ and $A_{\kappa}(y, e)=I_{e}^{y} e^{\lambda} F_{\kappa \lambda}(y)$. Notice the split-up into a string-independent part (the only one that would be present in the indefinite-metric approach in the Feynman gauge) and a string-dependent part.

With a hindsight from section 6 and 7 , we anticipate that the relevant contributions in the asymptotic limit arise only from the string-dependent parts of $D_{\kappa, \mu \nu}$ in $X_{1}$ and $X_{2}$, while the sum of all other contributions decays faster than $\lambda^{-2}$. We defer the proof of the latter statement to the end of the section.

In the relevant contributions, we partially integrate $\partial_{\kappa}^{y}$, using

$$
\partial_{\kappa}^{y} R\left(j^{\kappa}(y), \psi(f)\right)=-i f(y) \cdot \psi(y), \quad \partial_{\kappa}^{y} R\left(j^{\kappa}(y), \psi(f)^{*}\right)=i \overline{f(y)} \cdot \psi^{*}(y) .
$$

The boundary terms involving $\partial_{\kappa} g(y)$ can be roughly estimated to vanish in the adiabatic limit like $T^{-3}$ if the cutoff function $g(y)$ is chosen to drop from $q$ to 0 in the interval $T \leq\left|y^{0}\right| \leq T+1$. Specifically, the support of the retarded commutators is the backward lightcone of the support of $f$, and intersects the support of $\partial g$ in a strip of spatial volume 
$\sim T^{3}$. Taking into account the quadratic decay of two-point functions and propagators in timelike directions, the boundary terms are $\sim T^{3} / T^{6}$.

The bulk terms are

$i \int d^{4} y g(y)\left(f(y) \cdot\left(\partial_{y} \wedge e\right) I_{e}^{y} \Delta_{0}(y-x) \cdot\left\langle\psi(y) \psi(f)^{*}\right\rangle-\left\langle\psi(f) \psi^{*}(y)\right\rangle \cdot\left(\partial_{y} \wedge e\right) I_{e}^{y} \Delta_{0}(x-y) \cdot \overline{f(y)}\right)$.

In the adiabatic limit, we may assume that the support of $f$ is contained in the region where $g(y)=q$, and by the scaling behaviour $\lambda^{2} \Delta_{0}(\lambda x-y)=\Delta_{0}(x-y / \lambda)$ of the massless two-point function, we may simply neglect $y \in \operatorname{supp}(f)$ against $x$. We obtain in first order

$$
a(x)^{(1)}=\left.\lim _{\lambda \rightarrow \infty} \lambda^{2}\left\langle\psi(f) F(\lambda x) \psi(f)^{*}\right\rangle\right|_{g L(e)} ^{(1)}=q \cdot\left(\partial_{x} \wedge e\right) I_{-e}^{x} C_{0}(x) \cdot\left\langle\psi(f) \psi(f)^{*}\right\rangle,
$$

where $C_{0}$ is the massless commutator function. The last factor is $\|\Psi\|^{2}=1$. If we choose $e^{0}=0$, then $a(0, \vec{x})^{(1)}$ evaluated in the plane $x^{0}=0$ vanishes for the magnetic field, and

$$
\lim _{\lambda \rightarrow \infty} \lambda^{2}\left\langle\psi(f) \vec{E}(\lambda \vec{x}) \psi(f)^{*}\right\rangle=-q \cdot \vec{e} \int_{0}^{\infty} d s \delta(\vec{x}-s \vec{e}) .
$$

for the electric field. Thus the asymptotic electric field is concentrated at the direction $\vec{e}$ and points in the direction of $-\vec{e}$. The total flux is $-q$, as expected for the electron state $\Psi$, by the global Gauss Law, and in accord with section 4 . If $L(e)$ is averaged with a smearing function $h(e)$, the asymptotic flux density in the direction $\vec{x}=r \vec{e}$ is $-q h(e) / r^{2}$.

To show the vanishing of the sum of the remaining contributions $X_{3}$ and the stringindependent parts of $X_{1}, X_{2}$, we content ourselves with showing that their contribution to $\partial^{\mu}\left\langle\psi(f) F_{\mu \nu} \psi(f)^{*}\right\rangle$ is a sufficiently well-localized charge distribution of total charge zero, so its asymptotic field configuration vanishes. We use

$$
\begin{aligned}
\partial^{x \mu}\left(\partial_{\mu}^{y} \eta_{\nu \kappa}-\partial_{\nu}^{y} \eta_{\mu \kappa}\right) \Delta_{0} & =\left(-\eta_{\nu \kappa} \square^{y}+\partial_{\nu}^{y} \partial_{\kappa}^{y}\right) \Delta_{0}=\partial_{\nu}^{y} \partial_{\kappa}^{y} \Delta_{0}, \\
\partial^{x \mu} D_{\kappa, \mu \nu}^{y} G_{\mathrm{ret} 0} & =-\left(\eta_{\nu \kappa}+e_{\nu} I_{e}^{y} \partial_{\kappa}^{y}\right) \square^{y} G_{\mathrm{ret} 0}=-\left(\eta_{\nu \kappa}+e_{\nu} I_{e}^{y} \partial_{\kappa}^{y}\right) \delta(x-y),
\end{aligned}
$$

in the string-independent parts of $X_{1}$ and $X_{2}$, and in $X_{3}$, respectively.

Partial integration of $\partial_{\kappa}^{y}$ in the former produces bulk terms from the action on the propagators, as before, and boundary terms that identically cancel the corresponding contributions of the boundary terms in the string-dependent parts (because $-(e \partial)$ inverts the string-integration $I_{e}$ ). Partial integration of $\partial_{\kappa}^{y}$ in the latter produces only a boundary term that is identical with the rapidly decaying boundary term in (4.2).

Having settled the boundary terms, we collect the bulk terms:

$$
\begin{aligned}
q \cdot \int & d x_{1} d x_{2} f\left(x_{1}\right) \overline{f\left(x_{2}\right)} \partial_{\nu}^{x}\left(-i \Delta_{0}\left(x_{1}-x\right)+i \Delta_{0}\left(x-x_{2}\right)\right) \cdot\left\langle\psi\left(x_{1}\right) \psi^{*}\left(x_{2}\right)\right\rangle \\
& -g(x)\left\langle\psi(f) j_{\nu}(x) \psi(f)^{*}\right\rangle .
\end{aligned}
$$

For large $x$, one may again neglect $x_{i}$ in the arguments of $\Delta_{0}$. For $\nu=0$ and $x^{0}=0$, the first two terms combine into $\partial_{x}^{0} C_{0}(x)=\delta(\vec{x})$. The resulting contribution to the charge density in the asymptotic limit is

$$
\left\langle\psi(f)\left[q \delta(\vec{x})-g(x) j^{0}(0, \vec{x})\right] \psi(f)^{*}\right\rangle .
$$


This result exhibits a compensating point charge $q \delta(\vec{x})$ that one recognizes as coming from the "fictitious current". Indeed, its "position at $x=0$ " is fictitious because it is "seen from infinity". In the Feynman gauge calculation, the string-dependent part (5.4) would be absent and the total result would be (5.5).

The total charge in (5.5) vanishes in the adiabatic limit, hence the contribution to the asymptotic field configuration vanishes, too. Multi-pole radiation fields (that are not excluded by the vanishing total charge, and have a slower spatial decay) also have zero asymptotic field configurations, because the precise definition of the asymptotic limit [7] involves also an averaging in time that suppresses the oscillations.

\section{The hybrid approach}

The "hybrid approach" [26] allows to study the relation between the string-localized and the indefinite-metric approach. In particular, it sheds light on how the superselection structure of QED arises dynamically (section 7).

Let $A_{\mu}(x)$ be the usual point-localized vector potential, for simplicity in the Feynman gauge. In order to emphasize that it is defined only in a space with indefinite metric (Krein space, see appendix A.1), we denote it as $A_{\mu}^{K}(x)$. It can be decomposed as

$$
A_{\mu}^{K}(x)=A_{\mu}(x, e)-\partial_{\mu} \phi^{K}(x, e) .
$$

The string-localized potential $A_{\mu}(x, e)$, defined as the string integral (1.5) over the field strength, directly descends to the physical Hilbert space, whereas the massless "escort field"

$$
\phi^{K}(x, e):=\int_{0}^{\infty} d s A_{\mu}^{K}(x+s e) e^{\mu}
$$

lives on the Krein space. (The two parts of the operator $D_{\kappa, \mu \nu}$ in section 5 precisely correspond to $A_{\mu}^{K}(x)$ and $\partial_{\mu} \phi^{K}(x, e)$, respectively.)

The interaction density splits accordingly as

$$
L^{K}=A_{\mu}^{K} j^{\mu}=A_{\mu}(e) j^{\mu}-\partial_{\mu}\left[\phi^{K}(e) j^{\mu}\right] .
$$

$L(e)=A_{\mu}(e) j^{\mu}$ thus differs from $L^{K}$ by a total derivative that should be ineffective in the adiabatic limit. $L(e)$ is a priori defined on the Krein space, but descends to the physical Hilbert space, while the indefinite-metric degrees of freedom are "disposed of" with the discarded total derivative. We have checked up to second order [26] that the $S$ matrix with interaction density $g L^{K}-\partial_{\mu} g \cdot \phi^{K}(e) j^{\mu}$ coincides with the $S$ matrix with interaction $g L(e)$, i.e., the former descends to the Hilbert space where the latter is defined.

This pattern prevails in many models of interest: there is a string-independent pointlocalized interaction density $L^{p}$, possibly on a Krein space, such that $L(e)$ descends to the physical Hilbert space and $L^{p}-L(e)$ is a total derivative:

$$
L(e)=L^{p}+\partial_{\mu} V^{\mu}(e) .
$$

By definition, an interacting field is observable if and only if

$$
\left.\chi\right|_{g L(e)}=\left.\chi\right|_{g L^{p}-\partial g \cdot V(e)} .
$$


In particular, the left-hand side is defined on the Hilbert space and the right-hand side, in the adiabatic limit $\partial g=0$, does not depend on the string $e$ of the interaction density and is local because $L^{p}$ is point-localized, by the argument given in section 2 . By equality, $\left.\chi\right|_{g L(e)}$ enjoys both properties. This broad definition also includes cases like $\left.A\left(x, e^{\prime}\right)\right|_{L(e)}$ in QED, that satisfies (6.4) and hence is independent of $e$ and remains localized along the string $e^{\prime}$.

For the interacting Dirac field in QED, a remarkable formula is expected to hold:

$$
\left.\psi(x)\right|_{g L(e)}=: e^{i g \phi^{K}(x, e)} \psi(x):\left.\right|_{g L^{K}-\partial g \cdot j \phi^{K}} .
$$

(We have explicitly verified it up to second order [26]. In first order, it is equivalent to

$$
\int d^{4} y R\left(j^{\mu}(y), \psi(x)\right)\left[g(y)\left(A_{\mu}(y, e)-A_{\mu}^{K}(y)\right)+\partial_{\mu} g(y) \phi^{K}(y)\right]=i g(x) \phi^{K}(x, e) \psi(x),
$$

which is true by (6.1) upon partial integration, using (5.2).) The same holds if both $L(e)$ and $\phi^{K}(x, e)$ are smeared with a test function $h(e) .{ }^{4}$ Let us discuss its consequences.

The left-hand side is defined on the Hilbert space. Thus, it allows to define positive states on the algebra of the Maxwell field of the form $\omega(X)=\left(\Omega,\left.\left.\psi\right|_{g L(e)} X \psi\right|_{g L(e)} ^{*} \Omega\right)$ before the adiabatic limit $g(y) \rightarrow q$ is taken, and these states remain positive in the adiabatic limit. Thus, the interacting Dirac field complies with Hilbert space positivity.

On the other hand, $\left.\psi\right|_{g L(e)}$ is a priori badly delocalized in the adiabatic limit due to the string-localized interaction density (cf. section 2). But the right-hand side is manifestly string-localized (because with $\partial g=0, e$ appears only in the free field, and not in the interaction density). By equality, the interacting Dirac field is string-localized - without being just a string-integral over a point-localized field as (1.5).

String-localization is the best one may expect for the charged interacting Dirac field (cf. [9] for theories with a mass gap), and it is physically essential because string-localized fields do not commute with the asymptotic flux operators that measure the total charge [17]. It also secures enough causal separability for the needs of scattering theory.

Notice the "semi-perturbative" nature of (6.5), where the exponential already involves a partial summation in the coupling constant. It is this feature that allows to discern the emergence of superselection sectors in the next section.

The appearance of the exponential of the escort field on the right-hand side is also interesting in the context of the questions raised in [8]. Ignoring for the moment their singular nature, we note that operators like $\left.\left.\psi\right|_{q L(e)}\left(x_{1}\right) \psi^{*}\right|_{q L(e)}\left(x_{2}\right)$ involve, via (6.5),

$$
e^{i q\left(\phi^{K}\left(x_{1}, e\right)-\phi^{K}\left(x_{2}, e\right)\right)} \text {. }
$$

If (for simplicity) the string $e$ is chosen parallel to a straight line $\gamma$ from $x_{1}$ to $x_{2}$, it holds

$$
\int_{\gamma} d \vec{x} \cdot \vec{A}^{K}(\vec{x})=\phi^{K}\left(\vec{x}_{1}, e\right)-\phi^{K}\left(\vec{x}_{2}, e\right)
$$

\footnotetext{
${ }^{4}$ Since the free operator on the right-hand side of (6.5) is a special case of [22, eq. (2.6)] and [32, eq. (1.1)], the string-localized interaction on the left-hand side naturally implements these previous ideas of "QED in terms of gauge invariant fields".
} 
so that the operators (6.6) coincide with the unitaries ("gauge bridges") used in [8] to implement the local Gauss Law. In this guise, via the equality (6.5), the longitudinal degrees of freedom are "virtually present" in the string-localized QED on the Hilbert space. The next section puts these formal considerations onto a more solid ground.

\section{Photon cloud superselection}

The exponentials of the free escort field appearing in (6.5) are highly singular objects. We shall demonstrate how they can be regularized in such a way that (a) Hilbert space positivity is guaranteed (despite their original definition on the Krein space), and (b) they generate states with "photon clouds", and are therefore responsible for the uncountable superselection structure [6]. Thus, we believe them to be the "carriers" of the infinitely many asymptotic symmetries of the "infrared triangle" [34]. (The multiplying Dirac field in (6.5) plays no essential role in the argument and will be omitted from our simplified presentation.)

The photon clouds are characterized by the expectation values of asymptotic field operators as in section 5, that in turn define uncountably many superselection sectors. The method below was first used in a 1+1-dimensional model in order to understand the appearance of infra-particles [29]. Its application to QED is a new result. It shows along the way that the model was on the right track, even if its massless particles were not the photons of a gauge theory. The crucial fact is that the infrared state vectors do not exist in the original photon Fock space (tensored with the Dirac Fock space), but in a GNS Hilbert space reconstructed from the state functional, as it was also emphasized in Steinmann's approach [33] to QED.

The escort field itself is singular due to the logarithmic divergence of its two-point function. We first regularize it by introducing a mass $m$ :

$$
w_{m}\left(x-x^{\prime}, e, e^{\prime}\right)=\int_{H_{m}^{+}} d \mu_{m}(p) e^{-i p\left(x-x^{\prime}\right)} \frac{-e \cdot e^{\prime}}{(p \cdot e-i \varepsilon)\left(p \cdot e^{\prime}+i \varepsilon\right)},
$$

where $d \mu_{m}(p)=(2 \pi)^{-3} d^{4} p \delta\left(p^{2}-m^{2}\right) \theta\left(p^{0}\right)$. When the string directions are smeared with real test functions on the hyperboloid $e^{2}=-1$, one gets

$$
w_{m}\left(x-x^{\prime}, h, h^{\prime}\right)=\int_{H_{m}^{+}} d \mu_{m}(p) e^{-i p\left(x-x^{\prime}\right)}\left[-\overline{t(p, h)_{\mu}} t\left(p, h^{\prime \mu}\right]\right.
$$

where

$$
t(p, h)^{\mu}:=\int_{0}^{\infty} d s \int_{S^{2}} d \sigma(e) e^{i p \cdot s e} h(e) e^{\mu}=i \int_{S^{2}} d \sigma(e) \frac{h(e) e^{\mu}}{(p \cdot e)+i \varepsilon} .
$$

The positivity of $-\overline{t(p, h)_{\mu}} t(p, h)^{\mu}$ can be guaranteed by restricting the support of $h$ to the sphere $e=(0, \vec{e})$ (or any Lorentz transform of it), so that $t(p, h)=(0, \vec{t}(p, h))$, and $-\overline{t(p, h)_{\mu}} t(p, h)^{\mu}=|\vec{t}(p, h)|^{2} \geq 0$.

The massless limit of the distribution $w\left(z, h, h^{\prime}\right)$ is defined only for test functions $g(z)$ with $\int g(z) d^{4} z=\widehat{g}(0)=0$. In order to enable the massless limit for arbitrary test functions, 
we define

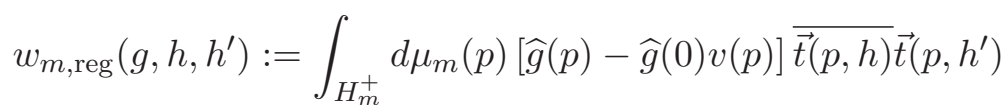

$$
\begin{aligned}
& =w_{m}\left(g, h, h^{\prime}\right)-\widehat{g}(0) \cdot c_{m, v}\left(h, h^{\prime}\right) \text {, }
\end{aligned}
$$

where $v$ is any test function with $v(0)=1$. A different choice of $v$ leads to an additive constant, and one can see that this is the only freedom of renormalization. On test functions with $\widehat{g}(0) \neq 0$, both $w_{m}\left(g, h, h^{\prime}\right)$ and $\widehat{g}(0) \cdot c_{m, v}\left(h, h^{\prime}\right)$ diverge in the massless limit, but their difference is finite. Due to the subtraction, $w_{m, \text { reg }}\left(x-x^{\prime}, h, h\right)$ is no longer positive.

We define the regularized exponential $e^{i q \phi^{K}(x, e)}$ of the escort field as

$$
V_{m}(f, h):=e^{-\frac{1}{2} q^{2} c_{m, v}(h, h)} \cdot: \exp i \phi_{m}^{K}(f, h):
$$

where the real test functions have total weights $\int d^{4} x f(x)=q$ and $\int_{S^{2}} d \sigma(\vec{e}) h(e)=1 . V_{m}$ is defined on the GNS Hilbert space of the positive two-point function $w_{m}\left(x-x^{\prime}, e, e^{\prime}\right)$. The massless limit can be taken as follows.

Let $g(z):=\int d y f(z+y) f(y)$, hence $\widehat{g}(0)=q^{2}$. By Wick's theorem,

$$
\begin{aligned}
\left\langle V_{m}(f, h) V_{m}\left(-f, h^{\prime}\right)\right\rangle & =\exp \left[w_{m}\left(g, h, h^{\prime}\right)-\frac{1}{2} q^{2} c_{m, v}(h, h)-\frac{1}{2} q^{2} c_{m, v}\left(h^{\prime}, h^{\prime}\right)\right]= \\
& =e^{i \alpha\left(g, h, h^{\prime}\right)} \exp \left[-\frac{1}{2} w_{m}\left(g, h-h^{\prime}, h-h^{\prime}\right)\right] e^{\frac{1}{2} w_{m, \operatorname{reg}}(g, h, h)+\frac{1}{2} w_{m, \mathrm{reg}}\left(g, h^{\prime}, h^{\prime}\right)},
\end{aligned}
$$

where $\alpha\left(g, h, h^{\prime}\right)=\operatorname{Im} w_{m}\left(g, h, h^{\prime}\right)$.

Because $\widehat{g}(0) \neq 0, w_{m}\left(g, h-h^{\prime}, h-h^{\prime}\right) \rightarrow+\infty$ diverges and consequently

$$
\left\langle V_{m}(f, h) V_{m}\left(-f, h^{\prime}\right)\right\rangle \rightarrow 0
$$

in the limit $m \rightarrow 0$, unless for all $p$

$$
\left|\vec{t}\left(p, h-h^{\prime}\right)\right|^{2}=0 .
$$

Write $\vec{H}(\vec{e})=\left(h-h^{\prime}\right)(0, \vec{e}) \vec{e}$. Then

$$
\frac{1}{(2 \pi)^{3}} \int d^{3} p e^{i \vec{p} \cdot r \vec{e}} \vec{t}(p, H)=\int_{S^{2}} d \sigma\left(\vec{e}^{\prime}\right) \int_{0}^{\infty} d s \delta\left(r \vec{e}-s \vec{e}^{\prime}\right) \vec{H}\left(\vec{e}^{\prime}\right)=r^{-2} \cdot \vec{H}(\vec{e}),
$$

and (7.4) is equivalent to $h^{\prime}=h$. Thus,

$$
\left\langle V(f, h) V\left(-f, h^{\prime}\right)\right\rangle= \begin{cases}0 & \text { if } h^{\prime} \neq h \\ \lim _{m \rightarrow 0} e^{w_{m, \mathrm{reg}}(g, h, h)}>0 & \text { if } h^{\prime}=h .\end{cases}
$$

As limits of states (after insertion of field operators), $\langle V(f, h) \ldots V(-f, h)\rangle$ are states on the free Maxwell field algebra. Because of the orthogonality (7.6), the GNS construction (cf. appendix A.2) yields uncountably many superselection sectors labelled by the directional smearing functions $h$, exhibiting the expected breakdown of Lorentz invariance [19], cf. section 1.2. 
We now compute the expectation values of the electromagnetic field strength in states of charge $-q$ implemented by the regularized exponentials $V(-f, h)$ of the free escort field. Again, we omit the multiplying Dirac field. By reproducing the same asymptotic field configurations determined by the directional smearing function $h$ as in section 5 , we see that only these exponential fields are responsible for the photon clouds.

In the sequel, $F$ and $\phi^{K}$ are free fields, hence their commutator is a multiple of $\mathbf{1}$. The state obtained from the vacuum by the adjoint action of $V(f, h)$

$$
\left\langle F_{\mu \nu}(x)\right\rangle_{f, h}:=\frac{\left\langle V(f, h) F_{\mu \nu}(x) V(-f, h)\right\rangle}{\langle V(f, h) V(-f, h)\rangle}=-i\left[F_{\mu \nu}(x), \phi^{K}(f, h)\right]
$$

differs from the vacuum by the automorphism $\beta(F)=F-i\left[F, \phi^{K}(f, h)\right]$. (The two terms of the commutator correspond to the asymptotic limits of the string-dependent parts of $X_{1}$ and $X_{2}$ in the calculation in section 5. The remaining terms that were shown in section 5 not to contribute asymptotically anyway, are absent here because we ignore the multiplying Dirac field.) The commutator is $\left.\left[F_{\mu \nu}(x), \phi^{K}(y, e)\right]=i\left(\partial^{x} \wedge e\right) I_{e}^{y} C_{0}(x-y)\right)$ in accord with (5.3), smeared with $f(y)$ and $h(e)$. Repeating the calculation after (5.3), gives the same result

$$
\lim _{r \rightarrow \infty} r^{2}\langle\vec{E}(r e)\rangle_{f, h}=-q \cdot h(e) \vec{e} .
$$

In other words, operators $V(f, h)$ with test functions $f(x)$ and $h(e)$ as specified, substitute the singular expression $e^{i q \phi^{K}(x, h)}$, that is only perturbatively defined in (6.5) (with $\left.\psi\right|_{L(h)}$ on the left-hand side). They yield charged states with the asymptotic electric flux density $-q h(e) / r^{2}$ in accord with the global Gauss Law, and with the first-order result (5.4) of full QED. Perturbing the Dirac field with different averages $L(h)=\int d \sigma(e) h(e) L(e)$, one can construct states with arbitrary "photon clouds" whose shape is given by the function $h$.

For a full treatment, the interacting Dirac field must be taken into account, and not only its "factor" $e^{i q \phi^{K}}$ in (6.5). It is clear from the above indirect construction of the exponential in a GNS representation, that (6.5) does not remain a tensor product in the interacting field: it is impossible to split "infrared matter" into charged particles and their photon clouds. This important algebraic message goes well beyond more formal treatments as in [34]. With a hindsight from the 2D model [29], it is expected that the presence of this "factor" will reduce the large-time fall-off below the kinematic LSZ falloff. As a consequence, and in accord with [36], cross sections with zero photon resolution vanish, and one has to resort to the Bloch-Nordsieck prescription (soft-photon inclusive cross sections, [2]). We hope to return to these issues in a separate paper.

Quite analogous results concerning the localization of the asymptotic flux and the inequivalence of representations, derived in an external field setting, have been reported in [14]. Interestingly, while in our approach the choice of the string-dependent interacting Dirac field creating the charged state is responsible for the string-dependent expectation value of the string-independent field operators, in [14] the string-dependence is attributed to the field operator itself, via the choice of an axial gauge condition in Dirac's quantization prescription. 


\section{Conclusions}

Gauss' Law is of eminent importance in QED. Its impact on the algebraic structure and the nature of charged particles seems to be the decisive feature that distinguishes (abelian) gauge theories [21].

We have presented several explicit perturbative calculations, showing that the construction of QED with the help of string-localized potentials does implement Gauss' Law in states with local charge distributions. In particular, the result in [8] that in QED (once it is constructed), string-localized potentials cannot be used for the implementation of "gauge bridges", is not an argument against the possibility of the perturbative construction of QED. To the contrary, Bogoliubov's formula applied to the string-localized interaction density implies Maxwell's equations and, by turning local free charged fields into string-localized interacting fields, resolves the well-known conflict [17] that the global Gauss Law cannot hold in a QED with local charged fields.

The mechanism rendering charged fields string-localized when coupled to stringlocalized potentials, while preserving the point-localization of observables, is perhaps the most interesting achievement of these potentials. It was not anticipated when they were originally proposed $[27,30,31]$ in order to improve the UV behaviour of perturbation theory. Their role is certainly not to provide the degrees of freedom necessary to construct charged states - which they cannot as emphasized by [8].

The string-localized potentials themselves appear only in the interaction density: the quantities of interest of the resulting theory are the local interacting field strengths (along with their fluxes) and the string-localized interacting matter fields (along with their pointlocalized currents), related by the interacting Maxwell equation (1.2).

We acknowledge that the paper [8] has made it clear that the quantum implementation of Gauss' Law requires degrees of freedom beyond the observables. We have presented a "hybrid formulation" that makes explicit the relation between the indefinite-metric and string-localized approaches. It reveals in particular how these degrees of freedom are "virtually present" (in the sense explained in section 6) in the guise of escort fields. Their role is to mediate between gauge theory and string-localized quantum field theory, by supplementing gauge theoretic observables with string-localized charge-carrying interpolating fields in a positivity-preserving way.

We have also shown that the approach satisfies Buchholz' infra-particle criterium (quadratic decay of the electric flux density) in first order, with the asymptotic field configuration specified by the direction of the string. Interestingly, what might seem to be just a gauge degree of freedom, becomes a feature of the charged state created by the Dirac field. Moreover, string-localized QED has an infrared mechanism (the 4D version of a mechanism first studied by one of us in a 2D model [29]) to understand the superselection structure of QED due to asymptotic photon clouds.

The perturbative construction of QED is not done on the observables separately. Instead, we perturb the Maxwell and Dirac fields simultaneously. The former, being observables, are then distinguished by remaining local under the string-localized perturbation, while the latter become genuinely string-localized. In this way, the dynamics of the theory 
itself "selects" the interacting observables (including the currents) in terms of their causal localization properties. Concerning the currents, the hybrid picture and (6.4) are the tool to establish the expectation that they are point-localized observables. The exponentiated escort fields in (6.5) intrinsically provide the "gauge bridges" that Brandt [5] constructed in terms of potentials with longitudinal degrees of freedom.

\section{Outlook}

We conclude with some remarks, tracing out the perspective that is expected to emerge from string-localized QFT (SLF) in more general theories.

Approaching a theory from different directions may better reveal its "inner makings". This is particularly worthwhile for gauge theories that hitherto seem to defy the framework of Local Quantum Physics [21].

SLF can be successfully applied to large classes of models beyond QED [31], including SM weak interactions [20]. We consider it as a promising alternative to the gauge theory plus BRST setting, that does not explicitly use indefinite metric at intermediate steps. In many important instances, it produces superficially the same results as gauge theory, e.g., comparing [20] with [15], but remains more economic (no ghosts) and physically transparent (e.g., massive vector bosons are massive from the outset). Its "hybrid" description (section 6) provides the laboratory necessary to recognize the relation (and hopefully equivalence) to the corresponding theory in the usual approach, and to explain the "miracle" why Gauge Theory despite its violation of positivity is so incredibly successful. Specifically, it allows to control the localization of the charged fields in QED. Through the exponentiation of the logarithmically divergent "escort field", it promises a new interpretation of the Bloch-Nordsieck prescriptions in scattering theory, by shifting the emphasis from momentum space to causal localization in spacetime.

In theories with massive vector particles, SLF allows to maintain renormalizability of the interaction with the Dirac current, because string-localized massive vector fields have a better short-distance behaviour than the Proca field. On the other hand, a version of the hybrid approach allows to use SLF to control positivity if massive QED is described by the renormalizable interaction with the indefinite massive Feynman gauge potential.

SLF extends gauge theory in the sense that gauge theoretical observables are complemented with gauge-invariant interpolating fields (in fact, in the hybrid approach this is literally what happens). They are subject to the same spectral analyticity properties of local QFT, and the fundamental Spin-Statistics and PCT Theorems also apply. This has the enormous benefit of providing a natural construction of particle states and scattering amplitudes for which the analytic on-shell properties are a consequence of causal separability of interpolating fields, as envisaged by the pioneers of dispersion relations in particle physics.

SLF becomes essential, and goes beyond the usual Lagrangean approaches, whenever particles of spin (or helicity) $\geq 1$ are involved. This fact is linked to the issue of symmetries [28] that is much less subtle when only $s=0$ or $s=\frac{1}{2}$ particles are present.

A prominent instance is "charge screening" in theories with massive vector bosons: the expectation value of the global charge operator associated with a local conserved current 
necessarily vanishes [35]. (Notice that this fact is at variance with the idea of "spontaneous symmetry breaking" which would require a divergent expectation value. $)^{5}$ It is presently not clear whether the charge screening effect extends to non-Abelian currents coupled to massive vector fields in SLF, so as to explain why Nature does not provide examples with exact particle multiplets (besides PCT). Specifically, as in gauge theory non-Abelian currents will not be gauge-invarint, they may not be local observables in SLF. This important question deserves further attention.

Thinking of SLF as an alternative to gauge theory, can only work if it is equally successful in determining the "correct" SM interactions. In contrast to interactions between particles of spin 0 or $\frac{1}{2}$, where the model-building physicist can choose interactions "at will", interactions involving particles of spin or helicity $>1$ are strongly constrained by causality and positivity. Indeed, in SLF with several massive or massless spin-one particles, like the electro-weak interactions, the Lie algebra structure arises not by a symmetry principle, but instead (via the principle of string-independence) as a consistency condition on the interaction density.

We expect that the new understanding of QED in terms of SLF will also shed new light on helicity 2: perturbative gravity. Specifically, SLF will provide a new look at the asymptotic (Bondi-Metzner-Sachs) symmetries at $h=2$ that avoids the NoGo theorems in point-local gauge theory.

SLF may eventually help to extend the conceptional framework of Axiomatic QFT, without giving away its roots in Locality and Positivity [21]. Especially Algebraic QFT is designed with the focus only on the observables, and regards charged fields as a useful device, that must (and can) be "added by hand" to conveniently describe charged sectors. In contrast, SLF, starting from a perturbation theory supplemented by a new underlying "principle of string-independence", first introduces free observables and charged fields on the same footing, and then bases the distinction between the interacting fields on the intrinsic difference in their causal localization properties. This difference is particularly instrumental in the case of the charge superselection rule.

\section{Acknowledgments}

We are indebted to the authors of [8], and especially to D. Buchholz, to whom this paper is dedicated, for insisting in the importance of "gauge bridges" for Gauss' Law in QED, and urging us to explain how we expect the issue to be solved in the string-localized approach. We thank J. Gracia-Bondía and P. Duch for valuable comments. JM has received financial support from the Brasilian research agency CNPq, and is also grateful to CAPES and Finep.

\section{A Some background material}

We compile some pertinent facts about quantum fields as operator-valued distributions. They may be obscured in the modern path integral treatments which encode algebraic

\footnotetext{
${ }^{5}$ This conflict with the terminology of the Higgs mechanism does not enjoy the attention that it deserves. The absence of SSB does not mean that there is no Higgs particle - only it is not the driving agent, but its presence is a necessity for the self-consistency of the theory, both in gauge theory [1] and in SLF [26, 31].
} 
properties like Hilbert spaces and commutation relations in a rather indirect way, and they fall outside the focus of purely fibre-bundle views of gauge theory that usually do not talk about operators at all. Along the way, we explain some arguments used in the main text.

\section{A.1 Vector potentials and indefinite metric}

Adding a gauge-fixing term $-\frac{\lambda}{2}(\partial A)^{2}$ to the QED Lagrangean results in the equations of motion

$$
\square A^{\nu}=(1-\lambda) \partial^{\nu}(\partial A)-q \bar{\psi} \gamma^{\nu} \psi,
$$

where $-q \bar{\psi} \gamma^{\nu} \psi=j_{\text {elm }}^{\nu}$ is the electromagnetic current. The field strength $F_{\mu \nu}=\partial_{\mu} A_{\nu}-$ $\partial_{\nu} A_{\mu}$ trivially satisfies the homogeneous Maxwell equation

$$
\partial_{\mu} F_{\nu \kappa}+\partial_{\nu} F_{\kappa \mu}+\partial_{\kappa} F_{\mu \nu}=0
$$

and, as a consequence of (A.1), the inhomogeneous equation

$$
\partial_{\mu} F^{\mu \nu}=j_{\text {fict }}^{\nu}+j_{\text {elm }}^{\nu}
$$

where the "fictitious current" $j_{\text {fict }}^{\nu}=-\lambda \partial^{\nu}(\partial A)$ on the right-hand side is present also without interaction $\left(q=0\right.$, hence $\left.j_{\text {elm }}^{\nu}=0\right)$. It prevents the validity of Gauss' Law, because the integral over the fictitious charge density is a non-vanishing operator. (In the Lorenz gauge $\lambda=\infty$, where $(\partial A)=0$ but $\square A_{\nu} \neq 0$, the right-hand side of (A.1) is not defined, and the fictitious current turns out to be $j_{\text {fict }}^{\nu}=\square A^{\nu}$ instead.)

The unique covariant two-point function of a free quantum field satisfying (A.1) with $q=0$ is

$$
\left(\Omega, A_{\mu}(x) A_{\kappa}(y) \Omega\right)=-\int \frac{d^{4} k}{(2 \pi)^{3}} \theta\left(k^{0}\right)\left(\eta_{\mu \kappa} \delta\left(k^{2}\right)+\left(1-\lambda^{-1}\right) k_{\mu} k_{\kappa} \delta^{\prime}\left(k^{2}\right)\right) e^{-i k(x-y)} .
$$

The two-point function determines the scalar product of states $|f\rangle=\int d^{4} x f^{\mu}(x) A_{\mu}(x) \Omega$ :

$$
\langle f \mid g\rangle=\int \frac{d^{4} k}{(2 \pi)^{3}} \theta\left(k^{0}\right)\left[-\eta_{\mu \kappa} \overline{\hat{f}^{\mu}(k)} \hat{g}^{\kappa}(k) \cdot \delta\left(k^{2}\right)-\left(1-\lambda^{-1}\right) \overline{(k \hat{f}(k))}(k \hat{g}(k)) \cdot \delta^{\prime}\left(k^{2}\right)\right] .
$$

These are obviously indefinite (there are states of negative norm-square) because of the presence of the Lorentz tensor and of the derivative $\delta^{\prime}\left(k^{2}\right)$ (the latter being absent in the Feynman gauge $\lambda=1)$. The indefinite-inner-product space generated by this field from the vacuum is called a "Krein space".

With the inner product (A.5), one finds four linearly independent states for each momentum, as opposed to the two physical photon polarization states. The unphysical states have to be eliminated by the Gupta-Bleuler or BRST method, defining the physical Hilbert space as a quotient space (a semi-definite subspace modulo the null states). States generated by the fictitious current have norm-square zero and are eliminated; but the vector potential is not defined on the quotient space.

The resulting two-point function of the field strength

$$
\left(\Omega, F_{\mu \nu}(x) F_{\kappa \lambda}(y) \Omega\right)=\int \frac{d^{4} k}{(2 \pi)^{3}} \delta\left(k^{2}\right) \theta\left(k^{0}\right) k_{[\mu} \eta_{\nu][\kappa} k_{\lambda]} e^{-i k(x-y)} .
$$


gives rise to a positive definite inner product, hence a Hilbert space. The subspace generated from the vacuum by the field strength is well-known to coincide with the physical quotient space, and it is the Fock space over the sum of the irreducible helicity \pm 1 Wigner representations (corresponding to the physical photon states).

In particular, the string-localized vector potential

$$
A_{\mu}(x, e):=\int_{0}^{\infty} d s F_{\mu \kappa}(x+s e) e^{\kappa}
$$

is defined on the physical Hilbert space. One easily computes, using the homogeneous Maxwell equation (A.2):

$\partial_{\mu} A_{\nu}(x, e)-\partial_{\nu} A_{\mu}(x, e)=-\int_{0}^{\infty} d s \partial_{\kappa} F_{\mu \nu}(x+s e) e^{\kappa}=-\int_{0}^{\infty} d s \frac{d}{d s} F_{\mu \nu}(x+s e)=F_{\mu \nu}(x)$.

On the other hand, the field strength is also defined on the indefinite Krein space where $A_{\mu}(x)$ is defined. On this space, the same string-localized potential (A.7) decomposes as

$$
A_{\mu}(x, e)=\int_{0}^{\infty} d s\left(\partial_{\mu} A_{\kappa}(x+s e)-\partial_{\kappa} A_{\mu}(x+s e)\right) e^{\kappa}=A_{\mu}(x)+\partial_{\mu} \phi(x, e)
$$

where the string-localized field $\phi(x, e):=\int_{0}^{\infty} d s A_{\kappa}(x+s e) e^{\kappa}$ is called "escort field". Only the sum of the two terms on the right-hand side descends to the physical Hilbert space, where the two terms are not seperately defined.

The hybrid approach of section 6 makes use of the decomposition (A.8) in the Krein space, in order to gain insight on the coupling of $A_{\mu}(x, e)$ to the Dirac field on the physical Hilbert space.

Distributional aspects. Strictly speaking, string-localized fields are distributions in both variables $x$ and $e$. Thus, they should be smeared with a test function $f(x, e)$ so as to produce well-defined operators. In the case of the QED interaction density $A_{\mu}(x, e) j^{\mu}(x)$, we choose $f(x, e)=g(x) h(e)$ and think of $h$ as an averaging with $\int d \sigma(e) h(e)=1$. The principle of string-independence requires that in the adiabatic limit $g(x) \rightarrow q$, the resulting interacting observables do not depend of the details of the function $h(e)$. In the case of the exponentiated escort field, the formal expression $e^{i q \phi(x, e)}$ is too singular to be considered without smearing. Thus, we smear the exponent with $f(x) h(e)$. Because of the "semiperturbative" nature of the hybrid approach, it is sufficient to study the exponential of the free escort field where the coupling constant appears in the exponent. Thus, we do not need a cutoff function $g(x)$ for the interaction density. Instead, we regard both $f$ and $h$ as averagings and absorb the coupling constant into the function $f$. This yields the conditions $\int d^{4} x f(x)=q$ and $\int d \sigma(e) h(e)=1$.

Massive case. In the massive case $\left(A_{\mu}=\right.$ Proca field), there is no problem with indefinite metric. The same formulas (A.7) and (A.8) define a string-localized potential and its escort field, both on the physical Hilbert space. The benefit of $A_{\mu}(x, e)$, as compared to the Proca field, is in this case the improved short-distance behaviour of the two-point function and the associated propagator, turning massive QED into a renormalizable theory $[25,30]$. Interestingly, the massless string-localized potential is a smooth limit of the massive stringlocalized potential [25], in marked contrast to the point-localized case. 


\section{A.2 Causality and observables}

Einstein Causality is the fundamental principle that two (experimentally realizable) operations on a system taking place at spacelike distance must not influence each other. Quantum field theory implements this principle by the postulate that the commutator of two observables localized at spacelike distance vanishes. Notice that only operators of observable quantities correspond to realizable operations of a system.

This makes of course no statement about non-observable quantities, but it implies that fields of half-integer spin that anti-commute at spacelike distance, cannot be observable. Only quadratic expressions such as currents can be observable.

The Spin-Statistics theorem is ofted said to state that quantum fields of half-integer spin must anti-commute at spacelike distance, and must commute with integer-spin fields. A closer look at the proof reveals that the correct statement is rather that half-integer spin fields cannot commute at spacelike distance, and the usual argument is only conclusive if no other options besides "commute" or "anti-commute" are allowed. This conclusion is in particular correct for free fields.

In string-localized QFT, "spacelike distance" means that the entire strings along which fields may be localized, are spacelike separated, cf. footnote 3.

Independent of the Spin-Statistics connection, charged fields in gauge theories are not observable because they are not gauge-invariant. Consequently, there is no apriori reason why they should obey any specific spacelike commutation properties at all, as long as their observable currents commute among themselves and with all other observables.

Gauss' Law, localization, and superselection sectors. The argument of [FPS] on QED shows that, if the global Gauss Law holds, the interacting Dirac field cannot commute like a point-localized field with the Maxwell field at spacelike distance. It can do so in the Krein space where the Gauss Law fails. The present work shows that in the Hilbert space the Dirac field becomes string-localized by the coupling to the vector potential, so that it still commutes with the Maxwell field in spacelike separation from the string. Because the string extends to infinity, it does not commute with the electric flux at infinity, and the conflict with Gauss' Law is resolved.

Because the electric field is observable, the limits

$$
\lim _{r \rightarrow \infty} r^{2} F^{0 i}(0, r \vec{e})
$$

("asymptotic flux densities" localized on the "sphere at spacelike infinity") commute with every local observable (but certainly not with string-localized quantities). By Schur's Lemma, they are multiples of $\mathbf{1}$ in every irreducible representation of the algebra of local observables. Different values of these operators characterize inequivalent representations (superselection sectors).

In section 5 , we determine their values in charged states by computing the expectation values $\left(\Psi, f^{0 i}(r \vec{e}) \Psi\right)$. If the charged states $\Psi$ are generated from the vacuum by interacting Dirac field operators, we find that the asymptotic flux density distribution has the same shape as the smearing function of the string direction used for the interaction. 
The "GNS construction" mentioned in section 7 assigns to a state (a positive expectation value functional on an algebra) a Hilbert space with a representation of that algebra in which the state is realized by a vector. Even if the functional is approximated by vectors in some Hilbert space, the resulting GNS Hilbert space may be unrelated to the Hilbert space used for the approximation. This happens in the case at hand, where the photon cloud superselection sectors are not realized in a Fock space.

Gauss' Law and infra-particles. As stated in section 5, weak convergence of the operator $\lim _{\lambda \rightarrow \infty} \lambda^{2} F_{\mu \nu}(\lambda x)$ for spacelike $x$ to a homogeneous function $a_{\mu \nu}(x)$ times the unit operator implies that charged particles cannot have a sharp mass [7], i.e., one-particle states cannot be eigenvectors of the mass operator. The (simplified) argument proceeds by observing that for eigenvectors $\Psi$ one has

$$
0=\left(\Psi,\left[P_{\kappa} P^{\kappa}, F_{\mu \nu}(x)\right] \Psi\right)=2 i\left(P^{\kappa} \Psi, \partial_{\kappa} F_{\mu \nu}(x) \Psi\right)-\left(\Psi, \square F_{\mu \nu}(x) \Psi\right) .
$$

In the asymptotic limit (1.4), $F_{\mu \nu}$ can be replaced by $a_{\mu \nu}$, and the second term decays more rapidly than the first, so that $\partial_{\kappa} a_{\mu \nu}(x) \cdot\left(P^{\kappa} \Psi, \Psi\right)$ must vanish. Because the scalar product here is generically non-zero, one concludes $\partial_{\kappa} a_{\mu \nu}(x)=0$ which by homogeneity entails $a_{\mu \nu}(x)=0$. Hence, $\Psi$ can be an eigenvector only if its total charge is zero.

Open Access. This article is distributed under the terms of the Creative Commons Attribution License (CC-BY 4.0), which permits any use, distribution and reproduction in any medium, provided the original author(s) and source are credited.

\section{References}

[1] A.W. Aste, G. Scharf and M. Dütsch, Perturbative gauge invariance: electroweak theory. II, Annalen Phys. 8 (1999) 389 [hep-th/9702053] [INSPIRE].

[2] F. Bloch and A. Nordsieck, Note on the radiation field of the electron, Phys. Rev. 52 (1937) 54 [INSPIRE].

[3] N.N. Bogoliubov, A.A. Logunov, A.I. Oksak and I.T. Todorov, General principles of quantum field theory, Kluwer, Dordrecht, The Netherlands (1990).

[4] N.N. Bogoliubov and D.V. Shirkov, Introduction to the theory of quantized fields, Wiley, New York, NY, U.S.A. (1959).

[5] R.A. Brandt, Field equations in quantum electrodynamics, Fortsch. Phys. 18 (1970) 249 [INSPIRE].

[6] D. Buchholz, The physical state space of quantum electrodynamics, Commun. Math. Phys. 85 (1982) 49 [inSPIRE].

[7] D. Buchholz, Gauss' law and the infraparticle problem, Phys. Lett. B 174 (1986) 331 [INSPIRE].

[8] D. Buchholz, F. Ciolli, G. Ruzzi and E. Vasselli, On string-localized potentials and gauge fields, Lett. Math. Phys. 109 (2019) 2601 [arXiv:1904.10055] [InSPIRE].

[9] D. Buchholz and K. Fredenhagen, Locality and the structure of particle states, Commun. Math. Phys. 84 (1982) 1 [INSPIRE]. 
[10] L.T. Cardoso, J. Mund and J.C. Várilly, String chopping and time-ordered products of linear string-localized quantum fields, Math. Phys. Anal. Geom. 21 (2018) 3 [arXiv:1709.03429] [INSPIRE].

[11] J.D. Dollard, Adiabatic switching in the Schrödinger theory of scattering, J. Math. Phys. 7 (1966) 802.

[12] P. Duch, Infrared problem in perturbative quantum field theory, arXiv: 1906.00940 [INSPIRE].

[13] M. Dütsch and K. Fredenhagen, Algebraic quantum field theory, perturbation theory and the loop expansion, Commun. Math. Phys. 219 (2001) 5 [hep-th/0001129] [INSPIRE].

[14] W. Dybalski and B. Wegener, Asymptotic charges, large gauge transformations and inequivalence of different gauges in external current QED, JHEP 11 (2019) 126 [arXiv: 1907.06750] [INSPIRE].

[15] M. Dütsch and G. Scharf, Perturbative gauge invariance: the electroweak theory, Annalen Phys. 8 (1999) 359 [INSPIRE].

[16] P.P. Kulish and L.D. Faddeev, Asymptotic conditions and infrared divergences in quantum electrodynamics, Theor. Math. Phys. 4 (1970) 745 [Teor. Mat. Fiz. 4 (1970) 153] [InSPIRE].

[17] R. Ferrari, L.E. Picasso and F. Strocchi, Some remarks on local operators in quantum electrodynamics, Commun. Math. Phys. 35 (1974) 25 [InSPIRE].

[18] W. Pauli and M. Fierz, Zur Theorie der Emission langwelliger Lichtquanten, Nuovo Cim. 15 (1938) 167.

[19] J. Fröhlich, G. Morchio and F. Strocchi, Charged sectors and scattering states in quantum electrodynamics, Annals Phys. 119 (1979) 241 [INSPIRE].

[20] J.M. Gracia-Bondía, J. Mund and J.C. Várilly, The chirality theorem, Ann. H. Poincaré 19 (2018) 843 [arXiv: 1702.03383] [InSPIRE].

[21] R. Haag, Local quantum physics, $2^{\text {nd }}$ ed., Springer, (1996).

[22] S. Mandelstam, Quantum electrodynamics without potentials, Annals Phys. 19 (1962) 1 [INSPIRE].

[23] G. Morchio and F. Strocchi, A nonperturbative approach to the infrared problem in QED: construction of charged states, Nucl. Phys. B 211 (1983) 471

[Erratum ibid. B 232 (1984) 547] [INSPIRE].

[24] G. Morchio and F. Strocchi, Infrared problem in QED and electric charge renormalization, Annals Phys. 168 (1986) 27 [INSPIRE].

[25] J. Mund, K.-H. Rehren and B. Schroer, Relations between positivity, localization and degrees of freedom: the Weinberg-Witten theorem and the van Dam-Veltman-Zakharov discontinuity, Phys. Lett. B 773 (2017) 625 [arXiv:1703.04408] [INSPIRE].

[26] J. Mund, K.-H. Rehren and B. Schroer, work in progress.

[27] J. Mund, B. Schroer and J. Yngvason, String-localized quantum fields and modular localization, Commun. Math. Phys. 268 (2006) 621 [math-ph/0511042] [INSPIRE].

[28] C.A. Orzalesi, Charges and generators of symmetry transformations in quantum field theory, Rev. Mod. Phys. 42 (1970) 381 [InSPIRE].

[29] B. Schroer, Infraparticles in quantum field theory, Fortsch. Phys. 11 (1963) 1 [InSPIRE]. 
[30] B. Schroer, An alternative to the gauge theoretic setting, Found. Phys. 41 (2011) 1543 [arXiv: 1012.0013] [INSPIRE].

[31] B. Schroer, The role of positivity and causality in interactions involving higher spin, Nucl. Phys. B 941 (2019) 91 [arXiv:1712.02346] [INSPIRE].

[32] O. Steinmann, Perturbative QED in terms of gauge invariant fields, Annals Phys. 157 (1984) 232 [InSPIRE].

[33] O. Steinmann, Perturbative quantum electrodynamics and axiomatic field theory, Springer, (2000).

[34] A. Strominger, Lectures on the infrared structure of gravity and gauge theory, Princeton University Press, U.S.A. (2018).

[35] J.A. Swieca, Charge screening and mass spectrum, Phys. Rev. D 13 (1976) 312 [InSPIRE].

[36] D.R. Yennie, S.C. Frautschi and H. Suura, The infrared divergence phenomena and high-energy processes, Annals Phys. 13 (1961) 379 [INSPIRE]. 